\title{
Hegel, la herida
}

\section{Hegel, the wound}

\author{
JESÚS EZQUERRA GÓMEZ \\ Universidad de Zaragoza
}

Recibido: 30/7/14 Aceptado: 29/01/15

\section{RESUMEN:}

La herida es el lógos, es decir, la identidad/diferencia entre algo y lo que ese algo es. El "entre". Esa relación (identidad/diferencia) separa en la misma medida que une y une en la misma medida que separa. Por eso escribe Hegel que "el conocer cura la herida que él mismo es" (Erkennen heilt die Wunde, die es selber ist). El silogismo en Hegel es el intento de restañar con el lógos la herida que el lógos es. Este ensayo también muestra cómo la concepción hegeliana del silogismo es anticipada por el "el más bello de los enlaces" (desmôn kállistos) del Timeo platónico y por el esquematismo kantiano.

PALABRAS CLAVE:

HEGEL, HERIDA, SILOGISMO, IDENTIDAD/DIFERENCIA, ESQUEMATISMO

\begin{abstract}
:
The logos could be regarded as a wound. The logos is the identity/difference between something and what this something is. The "between" itself. Such relation (identity/difference) separates to the same extent it unites, and it unites to the same extent it separates. For that reason Hegel writes: "Knowledge heals the wound that it itself is" (Erkennen heilt die Wunde, die es selber ist). The syllogism in Hegel is the attempt to stanch by means of the logos the wound that the logos itself is. This paper also shows how Hegel's concept of syllogism was previously suggested by the "fairest of all bonds" (desmôn kállistos) in the platonic Timeo and by Kantian schematism. KEYWORDS:

HEGEL, WOUND, SYLLOGISM, IDENTITY/DIFFERENCE, SCHEMATISM
\end{abstract}

(C) Contrastes. Revista Internacional de Filosofia, vol. XXI-Nº1 (2016), pp. 79-94. ISSN: 1136-4076

Departamento de Filosofía, Universidad de Málaga, Facultad de Filosofía y Letras Campus de Teatinos, E-29071 Málaga (España) 


\section{HERIDA}

EN EL PRINCIPIO ERA EL LÓGOS, es decir, la herida.

El verbo griego que corresponde al sustantivo lógos es légo. Su infinitivo -légein-significa recoger, recolectar, reunir, juntar. Con la expresión légein óstea, por ejemplo, designaban los griegos el acto de recoger los huesos del muerto después de la incineración tras haber apagado la pira con vino. ${ }^{1}$ De la misma raíz * leg deriva el verbo latino lego, que significa leer, pero también, en su primera acepción, recolectar, reunir, juntar (de donde legio: el contingente de soldados reclutados). ¿Qué recoge, reúne o junta el lógos? El lógos recoge, reúne o junta en un ente todo aquello que ese ente es (tomemos el ente Aquiles. lógoi serán: Aquiles es de pies ligeros, Aquiles es amigo de Patroclo, Aquiles es el matador de Héctor, et cetera). La reunión del ente con lo que es se muestra en el juicio, que es precisamente la atribución de algo (el ser) a algo (el ente). El algo al que se atribuye algo es el sujeto (Aquiles); el algo atribuido es el predicado (amigo de Patroclo) y la relación misma de atribución es la cópula (es). La estructura que subyace a todo juicio es, por lo tanto, ti katá tinós, es decir, «algo ( $t i)$ sobre, o acerca de (katá), algo (tinós)». No sólo a todo juicio -a todo légein- sino también a todo ser. Es tanto onto-lógica, como onto-lógica. El ser es justamente lo que junta o reúne, el katá, el respecto, el entre que hace posible que algo sea algo.

Ese reunir o juntar que los antiguos griegos denominaron lógos, delata una originaria escisión o diferencia. Sólo cabe, en efecto, reunir o juntar lo que no está unido, lo previamente disperso o disgregado. En alemán «juicio» se dice precisamente: Urteil, es decir, escisión (Teil: parte escindida) originaria (Ur-: prefijo que indica origen). ${ }^{2}$ Precisemos: no se alude con esta palabra a una primera escisión sino a que la escisión -la herida- es lo primero.

Esta escisión de lo unido o reunión de lo escindido es la relación, digámoslo ya, de identidad / diferencia entre un ente y su ser. Como dice el fragmento de Heráclito que en la edición de Diels-Kranz lleva el número 51, diapherómenon autò autôi symphéresthai («[lo uno, es decir, cada cosa] siendo diferente en sí mismo se identifica consigo mismo»). ${ }^{3}$ El lógos es el juego de symphérein / diaphérein; identidad / diferencia entre ser y ente. «Entre», es decir, herida. Conocer, en la medida en que tiene una estructura judicativa, es la expresión de una herida originaria.

1 Il. XXIII, 239 (en el caso de Patroclo) y Il. XXIV, 793 (en el caso de Héctor).

2 Véase el opúsculo de F. HöLDERLIN «Urteil und Sein», por ejemplo en F. Hölderlin, Werke und Briefe (Hg. Von F. Beisner \& J. Schmidt), Insel Verlag, Frankfurt a. M., 1969, t. II, pp. 591-592.

3 Este fragmento 51 de Heráclito es también citado, ligeramente modificado, en el Sofista 242e: diapherómenon gàr aeì sumphéretai («[el ser] siendo diferente es siempre idéntico»). 
Podríamos, por lo tanto, decir: la herida -el lógos- habla. Dice lo que las cosas son. Sin ella -sin él- las cosas no serían. Ser algo es estar herido, es decir, ser lógos, hablar. Esta es, a mi juicio, la idea fundamental de la filosofía de Hegel.

Aristóteles escribe en su Metafísica, recogiendo una idea de origen eleático: Tò mèn eînaí esti tò synkeîsthai kaì hèn eînai, tò dè mè eînai tò mè synkeîsthai allà pleío eînai (es decir: «el ser es el estar puesto conjuntamente y ser uno; el no ser, el no estar puesto conjuntamente sino el ser múltiple»). ${ }^{4}$

Esta es una idea que Aristóteles ha desarrollado en Metaphysica $\Gamma, 2$. ¿Por qué el ser es un «estar puesto conjuntamente», es decir, un com-puesto (syn-keîsthai)? El ser es para Aristóteles, en su primer y fundamental sentido, ousía, es decir, aquello de lo que decimos propiamente que es: tò òn. Pero de eso que es no decimos sin más que es, sino que es tal o cual cosa. Es decir, predicamos una pluralidad de atributos. Su ser, por lo tanto, es algo múltiple ( «lo que es se dice de muchas maneras $»^{5}$ ). Pero esos predicados no son una mera diversidad heterogénea. Están referidos a algo uno. Son unificados en el acto mismo de la predicación, convergen en aquello uno -la ousía - al que se atribuyen. Inhieren en él. La cópula -el «es»- los une a él. Por eso lo que es, es un uno ( $\left(\right.$ Lo uno no es algo diferente que lo que es» ${ }^{6}$ ).

Lo uno -escribe Pierre Aubenque- no es un predicado más entre otros: como en otros lugares muestra Aristóteles, lo uno es convertible con el ser, lo cual quiere decir que, cada vez que significamos el ser, significamos también la unidad. Cuando digo que Sócrates es hombre significo la unidad de Sócrates y la humanidad, o más bien la unidad de Sócrates dentro de la humanidad. Y en todos cuantos sentidos se diga el ser, en otros tantos significa la unidad: cuando digo «Sócrates es hombre», no significo la misma unidad entre sujeto y el predicado que cuando digo «Sócrates es enfermo». El problema de las significaciones del ser puede reconducirse, pues, sin inconvenientes al problema de las significaciones de lo uno, pues se trata del mismo problema. ${ }^{7}$

Todo juicio (lógos) tiene una estructura sintético-diairética. Es a la vez sýnthesis y diairesis, es decir, es un enlazar lo diverso que es al mismo tiempo un separar lo uno. ${ }^{8}$ Es una herida que se abre y que se cierra. Que habla.

$* * *$

4 Met. $\Theta 10,1051 \mathrm{~b} 11-13$

5 Véase Met. $\Gamma, 2$.

6 Met. Г, 2, 1003b 31-32.

7 P. Aubenque, El problema del ser en Aristóteles, Taurus, Madrid, 1981, pp. 154-155.

8 Véase sobre esto M. Heidegger, Logik. Die Frage nach der Wahrheit § 12, V. Klostermann, Frankfurt am Main, 1976, pp. 135-142. 
«El conocer - escribe Hegel en sus apuntes de clase sobre filosofía de la religión- cura la herida que él mismo es» (Erkennen heilt die Wunde, die es selber ist). ${ }^{9}$ Theodor W. Adorno repetirá una idea análoga, aplicándola específicamente a la filosofía, en su curso titulado Terminología filosófica: «La filosofía consiste en el esfuerzo del concepto por curar las heridas que necesariamente inflige el propio concepto». ${ }^{10}$ Más adelante, en el mismo curso, afirmará que «cada término filosófico es la cicatriz endurecida de un problema irresuelto». ${ }^{11}$ Lo que hiere es lo mismo que lo que cura. Sin embargo, la fuente de inspiración de Adorno no es, probablemente, Hegel sino Kierkegaard. En una carta a Krenek del 30 de septiembre de 1932 Adorno escribe refiriéndose al libro del filósofo danés Sygdommen til döden (La enfermedad mortal): «la enfermedad, dialécticamente es al mismo tiempo la cura». ${ }^{12}$

También en Nietzsche es central el tema de la herida. En Götzen-Dämmerung podemos leer:

Incluso en la herida continúa habiendo una fuerza curativa. Una sentencia, cuyo lugar de origen yo mantengo oculto a la curiosidad docta, viene siendo desde hace largo tiempo mi divisa: increscunt animi, virescit volnere virtus [se crecen los ánimos, se fortalece la fuerza con la herida]. ${ }^{13}$

Ciertamente no resulta extraño encontrarse con textos de esta naturaleza en Kierkegaard o Nietzsche. Pero ¿en Hegel? ¿Es Hegel un filósofo de la herida? ¿En qué sentido el conocer es para Hegel una herida? ¿Por qué y de qué modo la cura el propio conocer?

9 G.W.F. Hegel, Vorlesungsmanuscripte I, en G.W.F. HeGEL, Gesammente Werke, ed. crítica de la Rheinisch-Westfälischen Akademie der Wissenschaften en colaboración con la Deutschen Forschungsgemeinschaft, Felix Meiner, Hamburgo, 1968 y ss. (se citará en adelante esta edición con la abreviatura $G W$ ) t. XVII, p. 249 (trad. esp. de Ricardo Ferrara: G.W.F. Hegel, Lecciones sobre filosofia de la religión. 3. La religión consumada, Alianza Ed., Madrid, 1987, p. 42).

10 «Sie [i.e., die Philosophie] ist die Anstrengung des Begrifes, die Wunden zu heilen, die der Begriff notwendig schlagt», Тн.W. Adorno, Philosophische Terminologie I, 4 (clase del 17 de mayo de 1962), Suhrkamp, Frankfurt am Main, 1973, p. 55 (trad. esp. de R. Sánchez Ortiz de Urbina, en la ed. Taurus, Madrid, 1976, p. 45).

11 «Jeder philosophische Terminus die verhärtete Narbe eines ungelösten Problem sei», Tн.W. Adorno, Philosophische Terminologie II, 19 (clase del 6 de noviembre de 1962), Suhrkamp, Frankfurt am Main, 1974, pp. 10-11 (trad. esp. de R. Sánchez Ortiz de Urbina, en la ed. Taurus, Madrid, 1977, p. 10).

12 Ver S. Buck-Morss, Origen de la dialéctica negativa, s. XXI, Mexico, 1981, p. 92.

13 F. Nietzsche, Kritische Studienausgabe (ed. G. Colli \& M. Montinari), de Gruyter, Berlin / New York, 1967 ss., t. VI, p. 57. Tomo la traducción de A. Sánchez Pascual, (Alianza Ed., Madrid, 1975, pp. 27-28). El traductor nos desvela el origen de la divisa de Nietzsche: el poema de Furio de Anzio, Los Anales, transmitido por Aulo Gelio en sus Noches áticas, 18, 11, 4. 
La herida que se abre entre un ente y lo que ese ente es se dirime -se abre y se cura- en el juicio. Paradigma de la concepción judicativa del conocimiento en la modernidad es la filosofía kantiana. Kant es el filósofo de los límites y, por lo tanto, de aquello que une y separa. En esos límites ve Hegel heridas. Podríamos decir que con Hegel el límite es, no sólo en sí sino también para sí, herida.

\section{ENTRE EL SER Y EL SENTIDO}

En Kant el sujeto trascendental pone la cosa como lo que está (stellt) ante (vor) sí. Es decir, se la representa (vorstellt). La cosa es, por lo tanto, representación (Vorstellung). Ninguna representación es propiamente una cosa (en sí) sino un objeto, es decir, algo yecto ante mí, algo que se me objeta $(o b$-jekt $<$ ob-iectum). El sujeto es, por el contrario, aquella instancia ante la cual algo se presenta como objeto. Es decir, el sujeto, en tanto que aquello a lo que se objeta algo, es lo que confiere a este algo su objetividad. Es quien pone aquello que hace que algo sea un objeto. Pone, en suma, la objetividad del objeto. Dado que lo que hace que un objeto sea tal -es decir, un objeto- es el sujeto, podemos afirmar que este se erige en ser del ente. ${ }^{14}$ Las cosas en tanto que representaciones de un sujeto están fundadas ontológicamente por él. Así lo ha expresado Hegel en su Lógica:

El pensar elimina su [del objeto] inmediación, con él se presenta primeramente a nosotros, y lo convierte así en un ser-puesto, pero este ser-puesto suyo es su seren-sí y para sí, o sea su objetividad. El objeto es la unidad de la autoconciencia, en la que el objeto ha sido acogido; por lo tanto tiene esta objetividad en el concepto, y este es la unidad de la autoconciencia, en la que el objeto ha sido acogido; su objetividad, o sea el concepto, no es, por ende, otra cosa que la naturaleza de la autoconciencia, y no tiene otros momentos o determinaciones que el yo mismo. ${ }^{15}$

Dicho con la terminología escolástica: el concepto objetivo es generado por el concepto formal. ${ }^{16}$

Nuestros sentidos son asaltados incesantemente por multitud de sensaciones diversas. Sin embargo no percibimos una masa informe, indiferenciada de ellas: percibimos objetos. El objeto es algo unitario. Es, según Kant, una

14 Véase M. Heidegger, «Kants These über das Sein», en M. HeIDEgGer, Wegmarken, V. Klostermann, Frakfurt am Main, 1976, pp. 462-4.

15 G.W.F. Hegel, Wissenschaft der Logik (1816): GW XII, 18-19.

16 Véase X. Zubiri, Los problemas fundamentales de la metafísica occidental, Alianza Editorial, Madrid, 1995, pp. 256-8. Sobre los conceptos «objetivo» y «formal» véase F. SUAREZ, Disp. met. 2, sec. 1,1. 
unidad formal de diversas sensaciones, ${ }^{17}$ es decir, «aquello en cuyo concepto se halla unificado lo diverso de una intuición dada». ${ }^{18}$ Esta unidad, en tanto que formal, no puede venir dada por el lado de la sensación (la sensación es, por sí misma, mera diversidad, Mannigfaltigkeit) sino por el del sujeto trascendental. Este es, por lo tanto, quien pone en el objeto aquello que constituye su objetividad, es decir, su unidad. ¿Quién unifica la diversidad? El entendimiento (Verstand), que es para Kant facultad de juzgar. ${ }^{19}$ En el juicio se produce la síntesis por la que referimos un singular a un universal, una sensación a un concepto. Los conceptos son para Kant funciones de unidad de nuestras representaciones. ${ }^{20}$ Es decir, subsumen bajo sí una diversidad de sensaciones unificándolas, lo que los convierte en predicados de posibles juicios. La unificación de la diversidad sensible en un juicio es, por lo tanto, potestad del sujeto. Un juicio «no es -escribe Kant- más que la manera de reducir conocimientos dados a la unidad objetiva de la apercepción»». ${ }^{21} \mathrm{La}$ apercepción trascendental (lo que Hegel denominará la autoconciencia) es pues quien en última instancia hace posible la objetividad del objeto, es decir, el ser del ente.

Kant entiende el ser en términos judicativos, es decir, como lógos y, por consiguiente, transforma la ontología en lógica. ${ }^{22} \mathrm{El}$ su-jeto (sub-iectum) trascendental kantiano se constituye así como su-puesto (hypó-keímenon) ontológico de todo ente. Esta subjetivación de la vieja ousía aristotélica es la seña de identidad de la filosofía kantiana y de la modernidad filosófica.

Acabamos de caracterizar el proceder del entendimiento como aquél que subsume, mediante un juicio, un singular (intuición sensible) bajo un universal (concepto). Todo juicio que une un singular y un universal puede ser contemplado legítimamente como la conclusión de un silogismo que enlazaría tales términos por mediación de un particular. Hay, en efecto, un «término medio» en la analítica trascendental, que enlaza, como términos menor y mayor, a la sensación y al concepto: el esquema. Mutatis mutandis, el esquema juega en la economía trascendental el mismo papel que jugaba el término medio en el silogismo aristotélico (la misma palabra «esquema» evoca al silogismo, cuyas figuras eran denominadas por Aristóteles, justamente, «schêmata»). El esquema es para Kant una representación mediadora entre la sensación y el concepto. Media entre ambas instancias precisamente por su carácter jánico, es decir, por ser homogénea (gleichartig) tanto con la sensación como con el concepto. ${ }^{23} \mathrm{De}$

$17 \quad K r V$ A 105.

$18 K r V$ B 137.

19 Ibid.

20 KrV A 68/B 93.

$21 K r V$ B 141.

22 Véase G.W.F. Hegel, Wissenschaft der Logik [1832]: GW XXI, 35.

$23 K r V$ A 138/B 177 
hecho Kant lo denomina «concepto sensible» (sinnliche Begriff). ${ }^{24}$ Ese término medio descubre que la estructura silogística del conocimiento en Kant: Todo juicio (el respecto de un concepto a una sensación) resulta ser en realidad un silogismo: el respecto, mediado por un esquema (particular) de un concepto (universal) a una sensación (singular). Este término medio (lo racional) se halla en el juicio como algo implícito, latente, en la cópula, en el «es»». ${ }^{25}$ ¿Qué es lo que puede jugar ese papel de término medio (Mitte), siendo homogéneo tanto con la categoría como con la sensación? Aquello que es el medio (medium), el ámbito común de ambos en tanto que concepto integrador (Inbegriff) «en el que se hallan contenidas todas nuestras representaciones». Es decir, el tiempo. ${ }^{26}$ Por un lado, el carácter serial del tiempo -es decir, el que cada instante sea un momento de la misma serie- lo hace homogéneo con el concepto. Por otro, su diversidad -el que ningún instante se repita- lo hace homogéneo con la sensación. La serie temporal, unifica la diversidad de los instantes y, a la vez, el flujo temporal diversifica el ahora. ${ }^{27}$ Los esquemas son precisamente determinaciones temporales (Zeitbestimmungen), ${ }^{28}$ es decir, las formas o modos en los que el entendimiento intuitivo o imaginación ${ }^{29}$ determina el sentido interno. El esquema es, en rigor, la posición (pura) de un concepto. No se trata de que el esquema sea efecto (Wirkung) o determinación del entendimiento sobre la forma del sentido interno, es decir, sobre el tiempo, sino de que el entendimiento actúa sobre el sentido interno produciendo el tiempo esquemáticamente. Es decir, el tiempo no es algo sobre lo que obre la acción ponente del entendimiento, sino que es esa misma acción. Este es, creo, el sentido de la afirmación de Kant de que «el entendimiento no encuentra en el sentido interno semejante enlace de

$24 K r V$ A 146/B 186

25 G.W.F. Hegel, Glauben und Wissen; GW IV, 328-329. Véase también G. Jarczyk, Système et liberté dans la logique de Hegel, Aubier-Montaigne, París, 1980, pp. 89-91.

$26 K r V$ A 155/B 194.

27 Esta ambigüedad del tiempo ya fue señalada por Aristóteles en Phys. IV , 11219 b 12-15: «El ahora es por un lado como si fuera siempre el mismo, y por otro es como si no fuera nunca el mismo, pues en tanto que es cada vez en una cosa distinta, es cada vez otro (pues esto era en él el ser ahora), mientras que «lo que siendo en un momento dado» [el ahora] es el ahora, es lo mismo» (la traducción es mía), es decir, el ahora, serialmente considerado, es decir, en tanto que elemento en un orden de antero-posterioridad, es siempre lo mismo (es siempre un es entre un fue y un será), pero en lo relativo a lo que ahora es, es siempre otro (ahora es esto, ahora es esto otro...). Nuestra traducción se apoya en la interpretación de R. Brague en su ensayo «Sur la formule aristotélicienne ho pote ón (Physique, IV, 11 et 14)" recogido en su libro Du temps chez Platon et Aristote, PUF, Paris, 1982, pp. 97-144. Sobre este lugar en concreto véanse las pp. 99-104.

$28 K r V$ A 145/B 184.

29 Véase G.W.F. Hegel, Glauben und Wissen, GW IV, p. 341. 
lo diverso [e. d.: el tiempo], sino que lo produce afectándolo».$^{30}$ Este acto del entendimiento sobre la sensibilidad es el medium en el que están contenidos, como momentos suyos, la sensación y el concepto, y en el que, por lo tanto, adquieren propiamente sentido.

Más arriba hemos visto cómo el entendimiento unifica la diversidad sensible dada por la intuición en un juicio. La posición consistía por lo tanto en una acción sintética del entendimiento sobre la diversidad sensible. Sin embargo, esta acción, más allá de la actividad autónoma de una determinada facultad, sea el entendimiento u otra, apunta a una síntesis más originaria y radical que se vislumbra en el «entre»: entre la sensibilidad y el entendimiento o, como escribe Jean Hyppolite, entre el ser y el sentido:

El paso del uno al otro [e. d.: del ser al sentido] es la identidad originaria como mediación, el sentido se olvida y se pierde en el ser, pero el ser se ilumina como sentido. La conciencia sensible y el conocimiento son ese movimiento alternante del ser al sentido y del sentido al ser que presupone el germen originario, unidad de la unidad y de la diferencia del ser y del sentido. Que Hegel conciba ese germen como siendo él mismo mediación es el rasgo fundamental de su filosofía. Esta mediación ¿no está próxima a la temporalización; y sobre este punto no continúa Hegel a Kant? ${ }^{31}$

Esa acción de respectividad entre el entendimiento y la sensibilidad, previa a ambas facultades, es la síntesis imaginativa pura. ${ }^{32}$ Coincidimos, por consiguiente, con Heidegger en considerar radical (raíz común) y originario el papel de la imaginación en relación al entendimiento y a la sensibilidad. Sin embargo, no creemos que pueda ser, en rigor, considerada -como sugiere este filósofo- una facultad entendida como fuente específica de representaciones. ${ }^{33}$ Esta síntesis imaginativa es más bien un puro respecto entre facultades, su relative Identität.${ }^{34}$ En este sentido, es decir, en el de que no tiene una facultad

$30 K r V \mathrm{~B} 155$.

31 J. Hyppolite, «La critique hégélienne de la réflexion kantienne», en J. Hyppolite, Figures de la pensée philosophique I, PUF, Paris, 1971, pp. 186-7.

32 G.W.F. Hegel, Glauben und Wissen, GW IV, 327 y 341. Véase V. Vitiello, «Ethos/ Eros» II, 7, en V. Vitiello, La palabra hendida, Ediciones del Serbal, Barcelona, 1990, pp. 48-55.

33 M. Heidegger, Kant und das Problem der Metaphysik § 27, V. Klostermann, Frankfurt am Main, 1973, pp 134 ss.

34 G.W.F. Hegel, Glauben und Wissen, GW IV, 327 y 330 . Véase también J. Hyppolite, ob. cit., pp. 183 ss. Esta identidad relativa, en tanto que identidad de identidad y diferencia ( $G W$ IV, p. 327), es formalmente lo absoluto. Véase sobre esta concepción de lo absoluto: G.W.F. Hegel, Glauben und Wissen, GW IV, pp. 326-332, G.W.F. Hegel, Differenz des fichte'schen und schelling'schen Systems der Philosophie, GW IV, p. 64 y G.W.F. Hegel, Wissenschaft der Logik, Lehre vom Sein (1832), GW XXI, p. 60. 
propia que la sustente, es cierta la afirmación de Heidegger de que «la imaginación trascendental no tiene patria». ${ }^{35}$ Tal concepción apátrida de la imaginación es un rasgo característico del viraje dado por Kant en la segunda edición de la Crítica de la razón pura. En la primera edición (1781) la síntesis se origina en la imaginación, que es una facultad independiente del entendimiento y la sensibilidad. En la segunda, sin embargo (1787), tal síntesis es potestad del entendimiento (el entendimiento intuitivo) y, en última instancia, de la apercepción. ${ }^{36}$ Esta eliminación del carácter de facultad de la imaginación es sintomática de su nuevo lugar lógico: no es ya algo sustantivo, sino que es pura relación de respectividad. La imaginación es ahora únicamente el lugar de encuentro, el medium, o mejor el encuentro mismo, el «entre» que da sentido al juego de las facultades, cuya relación es. Éstas se revelan finalmente sólo como momentos de tal relación. Si alguna facultad correspondiese a la imaginación ésta sería la facultad de juzgar (Urteilskraft) en tanto que juego armónico de facultades, porque ella justamente

nunca consiste en una sola facultad, sino en su acuerdo, ya sea en un acuerdo determinado por una de ellas que desempeña un papel legislador [juicio determinante], ya sea más profundamente en un libre acuerdo indeterminado [juicio reflexionante]. ${ }^{37}$

Que el tiempo aparezca en Kant como respecto o posición entre facultades sólo es posible si la síntesis trascendental que lo constituye tiene, en última instancia, una naturaleza reflexiva. En efecto, el acto puro del entendimiento sobre la sensibilidad, en el que aquél pone (sintetiza) esquemáticamente la diversidad de ésta en y como tiempo, es autoafección de la mente: «espacio y tiempo -escribe Kant en el Opus postumum - son productos (pero productos primitivos) de nuestra propia imaginación; por tanto, intuiciones producidas en cuanto que el sujeto se afecta a si mismo". ${ }^{38}$ En la Kritik der reinen Vernunft se dice de la forma de la intuición -es decir, el tiempo-que

Al no representar más que lo puesto en la mente [Gemüt], no puede ser otra cosa que la manera según la cual la mente es afectada por su propia actividad,

35 M. Heidegger, op.cit. p. 136.

36 Ibid., § 31, pp. 163 ss.

37 G. Deleuze, Spinoza, Kant, Nietzsche, Labor, Barcelona, 1974, p. 180.. Una sugerente interpretación en esta línea hermenéutica puede verse en F. MARTínez MarzoA, Desconocida raíz común (estudio sobre la teoría kantiana de lo bello), Visor, Madrid, 1987.

38 I. Kant, Transición de los principios metafísicos de la ciencia natural a la física (Opus postumum), ed. de F. Duque, Editora Nacional, Madrid, 1983, p. 510. El subrayado es mío. Véase sobre la autoposición: pp. 476 ss. 
es decir [la actividad] de este poner su representación [ihrer Vorstellung], y por consiguiente [es afectada] por sí misma. ${ }^{39}$

Heidegger ha criticado la sustitución, propuesta por la edición de la obra completa de Kant de la Academia de Berlín en este texto, de «ihrer Vorstellung» («su representación», es decir, lo representado en la mente) por «seiner Vorstellung» («la representación de sí», es decir, la representación de la mente por esa misma mente). Lo que según Heidegger dice Kant es que «el representar puesto en la mente representa [vor-stellt] las 'relaciones puras' de la sucesión de la serie de los ahoras y las deja en manos de la receptividad». ${ }^{40}$ Sin embargo, la serie de los ahoras - el tiempo- no es algo dado, presentado ante la receptividad, sino que es el acto mismo de recepción de lo dado en la representación. El tiempo no es lo constitutivo de lo representado per se, sino que es algo más originario: es el acto de afección del sentido interno en virtud del cual toda representación se constituye como tal. El tiempo, por consiguiente, no es, por así decir, «recibido» de fuera sino que es producido por la propia receptividad en el acto de recepción de lo representado. El tiempo es el punto en el que receptividad y espontaneidad coinciden y esto es lo que hace de él el centro de la analítica trascendental. La representación que sirve de estribo, punto de partida o presupuesto para la autoafección que es el tiempo no es, por lo tanto, algo exterior en el sentido de un «afuera» originario, sino que está constituida por esa misma autoafección. Es decir, la posición de su (ihrer) representación en que consiste tal autoafección es en última instancia posición de la representación de sí (seiner). No me parece por lo tanto acertado caracterizar la autoafección constitutiva de la subjetividad en Kant - como ha hecho, por ejemplo, Gilles Deleuze- como «pliegue», pues todo pliegue presupone una trascendencia, es decir, un afuera originario que se invagina (se pliega) creando un espacio interior, un «adentro». Este no es el caso de la subjetividad en Kant. En él lo originario no es el «afuera», si es que se puede siquiera hablar aquí de tal, sino el acto reflexivo sintético puro del yo: el tiempo. Este, escribe acertadamente G. Böhme, «vale siempre para Kant como lo más originario». ${ }^{41}$ La autoafección kantiana no es pliegue sino reflexión.

Esta autoposición originaria, acto sintético reflexivo puro de la imaginación (el tiempo) es la raíz común de la que brotan, como momentos suyos, la receptividad y la espontaneidad; la sensibilidad y el entendimiento; la intuición y el concepto; la conciencia empírica y la conciencia pura; el objeto y el sujeto; el no-yo y el yo.

$39 K r V$ B 67-8.

40 M. Heidegger, Kant und das Problem der Metaphysik § 34, nota 266, ed. cit., p. 191.

41 G. Böhme, Zeit und Zahl. Studien zur Zeittheorie bei Platon, Aristoteles, Leibniz und Kant, V. Klostermann, Frankfurt am Main, 1974, p. 272. 
Pero todo acto de sintesis, por originario que sea, presupone una diaíresis, una diferencia, una distancia, una herida.

\section{El MÁS BELLO DE LOS VÍNCULOS}

Según Timeo ${ }^{42}$ lo generado por el demiurgo, dado que ha de ser visible (horatós) y tangible (haptós), ha de estar constituido por el fuego (que hace visible) y la tierra (que hace tangible). Pero la síntesis de estos dos elementos es posible por la mediación de un tercero que se halla en una relación de analogía con los extremos tal que

No es posible unir bien dos elementos aislados sin un tercero, ya que es necesario un vínculo en el medio que los una. El vínculo más bello es aquél que puede lograr que él mismo y los elementos por él vinculados alcancen el mayor grado posible de unidad. La proporción es la que por naturaleza realiza esto de la manera más perfecta. En efecto, cuando de tres números cualesquiera, sean enteros o cuadrados, el término medio es tal que la relación que tiene el primer extremo con él, la tiene él con el segundo, y, a la inversa, la que tiene el segundo extremo con el término medio, la tiene este con el primero; entonces, puesto que el medio se ha convertido en principio y fin, y el principio y fin, en medio, sucederá necesariamente que así todos son lo mismo y, al convertirse en idénticos unos a otros, todos serán uno. ${ }^{43}$

Este es según Platón el más bello de los vínculos (desmôn kállistos). ${ }^{44}$ En este texto veía Hegel un antecedente de su propia concepción del silogismo racional (Vernunftschluss) como movimiento reflexivo de identidad / diferencia que constituye la subjetividad (absoluta). ${ }^{45}$ Ya en su Differenz-Schrift de 1800 invocaba Hegel este pasaje platónico para explicar la «oposición real» de sujeto y objeto por la que «la realidad de los opuestos y la oposición real se produce sólo mediante la identidad de ambos». ${ }^{46}$ Hegel va más allá que Platón al aplicar este movimiento silogístico a la trinidad sistemática lógicanaturaleza-espíritu. En las anteriormente mencionadas Vorlesungen Hegel lo explica con lenguaje teológico:

42 Tim. $31 \mathrm{~b}$ ss.

43 Tim. 31 c 2-32 a 7. Reproduzco la traducción de F. Lisi (Gredos, Madrid, 1992).

44 Tim. $31 \mathrm{c} 2$.

45 Véase G.W.F. Hegel, Vorlesungen über die Geschichte der Philosophie II, en G.W.F. Hegel, Werke (ed. de E. Moldenhauer \& K. M. Michel), Suhrkamp, Frankfurt am Main, 1971, t. XIX, pp. 89-91.

46 Véase G.W.F. Hegel, Differenz..., GW IV, p. 65. 
Dios se ha hecho sujeto, lo que quiere decir que crea a su hijo, el mundo, se realiza en esta realidad, que aparece como otro, pero en la que permanece idéntico consigo mismo, aniquila la caída [Abfall] y se une conclusivamente [sich zusammenschliesst] en lo otro sólo con sigo mismo; así él es en primer lugar espíritu. ${ }^{47}$

Es decir, el «bello vínculo» platónico describe justamente para Hegel el movimiento espiritual que une y escinde (identifica/diferencia) a la lógica (Dios) y a la naturaleza por mediación del Cristo (que es amor). Dios para Hegel es lógos: un silogismo («Gott ein Schluss ist»), ${ }^{48}$ pero un silogismo «der sich mit sich selbst zusammenschliesst». ${ }^{49}$ Es decir, un silogismo en cuyo segundo momento se diferencia de sí -se enajena, se hace lo otro de sí (es decir, mundo)- y en cuyo tercer y último momento, por medio del término medio (el Cristo), el principio (Dios) se reune conclusivamente con el fin (la naturaleza) y se reconoce como lo mismo que él. Por lo tanto Hegel aplica el desmós kalós -que en principio se limita en el Timeo a la síntesis de los cuatro elementos- a la dialéctica entre to autó (Dios, la lógica) y to héteron (la naturaleza) haciendo una suerte de reconstrucción racional del mito platónico.

El elemento clave en una estructura de tipo silogístico es el término medio. Este, en virtud de su carácter jánico (es término mayor respecto al menor y menor respecto al mayor), es el que permite la síntesis de los extremos, la identidad de los diferentes. Él es el que hace que los términos de la estructura silogística sean «todos lo mismo». A este término medio lo llama Platón en el Banquete «Eros». Eros, estando en medio (en mésōi) entre hombres y dioses, llena el hueco entre ambos de tal modo que «el todo queda ligado consigo mismo» (tò pân auto autôi syndedésthai). ${ }^{50}$ Eros (Amor), en virtud de su carácter intermediario, hace posible el sýndesmos o enlace del todo consigo mismo. Aquello que hace posible que sea, justamente, un todo y no un mero agregado disperso y caótico. Como ha escrito con acierto Giovanni Reale, Amor es una auténtica copula mundi. ${ }^{51}$ Tiene, por consiguiente, una función análoga al vínculo bello del Timeo. ${ }^{52}$

Pero el amor es también herida. Eros tiene el doble carácter de unir lo separado y dividir lo uno:

47 G.W.F. Hegel, Vorlesungen über die Geschichte der Philosophie II, ed. cit., t. XIX, p. 91.

48 Ibid.

49 Ibid.

50 Symp. 202 e 6-7.

51 G. Reale, Eros, demonio mediador, Herder, Barcelona, 2004, p. 179.

52 Coincido en esto con G. Reale. Véase ob. cit., pp. 190-191. Ver también G. Reale, Por una nueva interpretación de Platón, Herder, Barcelona, 2003, pp. 657-675. 
El poder generador y demiúrgico de Eros parece, pues, desdoblarse en dos aspectos complementarios: es al mismo tiempo unificador y diferenciador. De la división generada por la unión nace un orden en el que, para retomar la idea neoplatónica, se armonizan las diferencias. ${ }^{53}$

\section{Vinzenzo Vitiello ha escrito que}

La filosofía tiene en Hegel la función erótica de reunificar lo hendido. ¿Hendido por qué? Por el entendimiento. El entendimiento es la potencia que niega, que separa y escinde el absoluto, la unidad, en opuestas multiplicidades: sentido y razón, sujeto y objeto, finito e infinito, amor profano y amor sacro... El entendimiento sin embargo, precisamente en cuanto que separa, está ligado al absoluto. Como el rostro del andrógino hendido, está dirigido a mirar el corte y por consiguiente a mirar también de alguna manera más allá de este. ${ }^{54}$

¿Qué es el término medio? En rigor, nada. Es pura relación de respectividad: término mayor respecto al menor y menor respecto al mayor. No tiene, pues, subsistencia. Es lo que debe desaparecer en una cosa para que sea lo que es. La diferencia, la herida. Como escribe Gwendoline Jarczyk el término medio es «el acto de pasar de lo que pasa». ${ }^{55}$ Es aquello inexistente en virtud de lo cual todo existe. ${ }^{56}$

En el contexto de la Lógica el término medio es la esencia; en el contexto del sistema el término medio es la lógica. La esencia, en efecto no es para Hegel algo que sustente la apariencia, que salvaguarde su sentido, su razón, es decir, aquello que hace que sea lo que es y no otra cosa. No. La esencia es aquello que desaparece, que se hunde, que se abisma, para que lo que existe aparezca tal cual es. Dicho de otro modo: la razón de algo, su qué es, se resuelve en su que es. El dispositivo que pone a punto en su lógica el gran racionalista que es Hegel, es, paradójicamente, la desaparición de la lógica; es decir, la muerte de Dios. Decir que la lógica es el pensamiento de Dios antes de la creación del mundo y de un espíritu finito ${ }^{57}$ es como decir que la esencia del mundo y del espíritu es Dios, pero un Dios que ha muerto, un Dios que no es sino el acto de pasar de lo que pasa.

Al principio del tercer capítulo de la primera sección de la Doctrina del concepto de la Ciencia de la lógica, Hegel nos dice que el silogismo (Schluss)

53 C. Calame, Eros en la antigua Grecia, Akal, Madrid, 2002, p. 186.

54 V. Vitiello, ob. cit., p. 21.

55 G. Jarczyk, «Médiation hegelienne, réalité de notre culture», en G. JARCZYK \& P.-J. Labarrière, Hegeliana, PUF, Paris, 1986, p. 40.

56 G. Jarczyk, «La médiation réflexive», en G. JARCZYK \& P.-J. LABARRIĖRE, ob. cit., p. 53.

57 G.W.F. Hegel, Wissenschaft der Logik, die Lehre vom Sein, Einleitung, GW XXI, p. 34. 
es la restauración (Wiederherstellung) del concepto (Begriff) en el juicio (Urtheil)..$^{58}$

El concepto se presentaba como una unidad. Tenía sus momentos (Momente) superados y conservados (aufgehobene) en esa unidad. En el juicio, por el contrario, esos momentos estaban, aunque relacionados entre sí, puestos como extremos independientes (selbstständige Extreme). Finalmente, en el silogismo la unidad conceptual es restaurada, si bien mediada por la escisión judicativa. El silogismo es la restauración del concepto, pero no desde el juicio sino en el juicio, en la escisión originaria (im Urtheile). En esta restauración, el juicio y su escisión no quedan atrás sino que permanecen en ella. El silogismo es, por decirlo con una expresión que utiliza Hegel en el prólogo de la Fenomenología del espíritu para caracterizar el saber: «la pura igualdad consigo mismo en el ser otro». .59

Esta unidad, en tanto que retornada, restaurada, ya no es inmediata sino puesta. El silogismo es, por lo tanto, el concepto completamente puesto (der vollständig gesetzte Begriff), ${ }^{60}$ es decir, una identidad de la identidad y la no identidad; una identidad no abstracta sino, por así decir, preñada de diferencia. En eso radica la racionalidad. Por eso el silogismo es lo racional y todo lo racional es un silogismo. ${ }^{61}$ Lo racional en Hegel no anula lo real, no lo sobrevuela. Lo racional es lo real mismo en toda su crudeza, complejidad e irreductibilidad. En toda: sin dejar nada fuera. Apurando el cáliz de su pasión y muerte, haciéndolo suyo. Como escribe Hegel en uno de los textos más bellos y potentes de toda su obra:

La vida del espíritu no es la vida que se espanta ante la muerte y se preserva pura de la desolación, sino la que la soporta y se mantiene en ella. El espíritu sólo alcanza su verdad en la medida en que se halla a sí mismo en el absoluto desgarramiento. ${ }^{62}$

El espíritu es eso: La vida que encierra en su seno la muerte; el orden que incluye en sí el caos; la belleza que anuncia lo terrible (Rilke); el amor cuyo envés es el odio; la interioridad que es puro afuera, el cierre conclusivo (Schluss) que es apertura, el cuerpo que no es sino todas sus heridas...

58 G.W.F. Hegel, Wissenschaft der Logik, die Lehre vom Begriff, GW XII, p. 90.

59 G.W.F. Hegel, Phänomenologie des Geistes, GW IX, p. 40.

60 G.W.F. Hegel, Wissenschaft der Logik, die Lehre vom Begriff, GW XII, p. 90.

61 Ibid.

62 «Aber nicht das Leben, das sich vor dem Tode scheut und von der Verwüstung rein bewarhrt, sondern das ihn erträgt, und in ihm sich erhält, ist das Leben des Geistes. Er gewinnt seine Wahrheit nur, indem er in der absoluten Zerrisenheit sich selbst findet». G.W.F. Hegel, Phänomenologie des Geistes, GW IX, p. 27. 
Decir que «todo es silogismo» es como decir que todo ha de salir de sí y reconocerse en ese afuera para encontrarse, en verdad, a sí mismo. Ese afuera -la herida - no queda en Hegel sin más restañado, curado, anulado. Ese afuera es lo que da sentido a la interioridad, a la vida. Es el fundamento de lo que hay. Un fundamento (Grund), empero, que no es, finalmente, sino abismo (Abgrund).

JESÚS EZQUERRA GÓMEZ es Doctor en filosofía por la Universidad Autónoma de Madrid con una tesis sobre Hegel («Tiempo y razón en Hegel»,1994). Profesor de Enseñanza Secundaria entre 1998 y 2007. Desde 2007 imparte docencia en el área de Ética y Filosofía Política en la Universidad de Zaragoza.

\section{Líneas de investigación:}

Se ha centrado principalmente en la relación entre ontología y política. Se ha ocupado de autores como Spinoza, Hegel, Miguel de Molinos o Sade.

Publicaciones:

Un claro laberinto. Lectura de Spinoza, Prensas de la Universidad de Zaragoza, Zaragoza, 2014.

El profundo de la nada (el desapego de Dios en el místico aragonés Miguel de Molinos), Institución Fernando el Católico (CSIC), Zaragoza, 2014.

Correo electrónico: jesusezq@unizar.es 
